\title{
Etude Comparative De La Rentabilité Des Systèmes De Pompage Solaire Et Thermique Sur Le Périmètre Irrigué De Soumarana Au Niger
}

\section{Illiassou Naroua,}

Département des Sciences de l’Environnement, Faculté des Sciences

Agronomiques, Université Boubakar Bâ de Tillabéri, Niger

\section{Abdoulkadri Laouali,}

Département de l’Agro-Socio-Economie, Faculté des Sciences

Agronomiques, Université Boubakar Bâ de Tillabéri, Niger

Souley Issaka,

Département de Production Durable des Cultures, Faculté des Sciences

Agronomiques, Université Boubakar Bâ de Tillabéri, Niger

Maman Rachid Salissou Issoufou,

Université Boubakar Bâ de Tillabéri, Niger

Hassoumi Djibo,

Département de l'Agro-Socio-Economie, Faculté des Sciences

Agronomiques, Université Boubakar Bâ de Tillabéri, Niger

Doi:10.19044/esj.2021.v17n43p246

Submitted: 09 December 2021

Accepted: 28 December 2021

Published: 31 December 2021
Copyright 2021 Author(s)

Under Creative Commons BY-NC-ND 4.0 OPEN ACCESS

Cite As:

Naroua I.,Laouali A., Issaka S., Issoufou M. R.S., \& Djibo H.,(2021). Etude Comparative De La Rentabilité Des Systèmes De Pompage Solaire Et Thermique Sur Le Périmètre Irrigué De Soumarana Au Niger European Scientific Journal, ESJ, 17(43), 1.

https://doi.org/10.19044/esj.2021.v17n43p246

\section{Résumé}

Le Niger dispose d'important potentiel de terre irrigable et plusieurs formes d'irrigation ont été développées notamment la petite irrigation, afin de réduire la dépendance de la production vis-à-vis des aléas climatiques. L’objectif de ce travail est de justifier le besoin de vulgariser la technologie des équipements de pompage solaire. Pour cela une enquête technicoéconomique a été conduite auprès d'un échantillon de 80 exploitants choisis de manière aléatoire sur le périmètre irrigué de Soumarana, à raison de 40 irrigants utilisant des systèmes de pompage thermique et 40 des systèmes de pompage photovoltaïque. Les cultures de carotte et d'oignon, étant les principales spéculations, ont été 
retenues pour conduire l'étude afin de comparer les performances de systèmes de pompage en place. Il ressort de l'analyse des résultats que le système de pompage solaire dégage des marges brutes nettement supérieure à celles du système de pompage thermique avec des différences de l’ordre de 402148 FCFA/ha et 342812 FCFA/ha respectivement pour la culture de carotte et celle de l'oignon. De même, le coût du pompage du mètre cube d'eau en système de pompage solaire est nettement inférieur à celui du système de pompage thermique avec respectivement $57,27 \mathrm{FCFA} / \mathrm{m}^{3}$ et $80,75 \mathrm{FCFA} / \mathrm{m}^{3}$. Ces résultats indiquent une meilleure performance du système de pompage solaire comparativement au système thermique.

Mots clés: Pompage Solaire, Pompage thermique, Rentabilité Economique, Soumarana.

\title{
Comparative Study of The Profitability of Solar and Fuel Powered Pumping Systems on the Soumarana Irrigated Perimeter in Niger
}

\section{Illiassou Naroua,}

Département des Sciences de l'Environnement, Faculté des Sciences

Agronomiques, Université Boubakar Bâ de Tillabéri, Niger

\section{Abdoulkadri Laouali,}

Département de l’Agro-Socio-Economie, Faculté des Sciences

Agronomiques, Université Boubakar Bâ de Tillabéri, Niger

Souley Issaka,

Département de Production Durable des Cultures, Faculté des Sciences

Agronomiques, Université Boubakar Bâ de Tillabéri, Niger

\section{Maman Rachid Salissou Issoufou,}

Université Boubakar Bâ de Tillabéri, Niger

\section{Hassoumi Djibo,}

Département de l’Agro-Socio-Economie, Faculté des Sciences

Agronomiques, Université Boubakar Bâ de Tillabéri, Niger

\begin{abstract}
Niger Republic has significant potential for irrigable land and several forms of irrigation have been developed, including small-scale irrigation, in order to reduce the dependence of production on climatic hazards. The objective of this study is to justify the need to popularize solar pumping equipment technology. To this purpose, a techno-economic survey was conducted among a sample of 80 farmers chosen randomly in the Soumarana irrigated area, at the rate of 40 farmers under fuel powered pumping system
\end{abstract}


and 40 under photovoltaic pumping system. Carrot and onion crops, being the main speculations, were selected to conduct the study to compare both pumping systems performances. The analysis of the results shows that the solar pumping system has higher gross margins than the fuel powered pumping system with differences of the order of 402148 FCFA/ha and 342 812 FCFA/ha respectively for carrot and onion crops. Similarly, the cost of pumping per cubic meter in the solar pumping system was clearly lower than that of the fuel powered pumping system with respectively $57.27 \mathrm{FCFA} / \mathrm{m}^{3}$ and $80.75 \mathrm{FCFA} / \mathrm{m}^{3}$. These results indicate a better performance of the solar pumping system compared to the fuel powered ones.

Keywords: Solar pumping, Fuel powered pumping, Economic profitability, Soumarana

\section{Introduction}

$\mathrm{Au}$ Niger, l'économie est tributaire du secteur agricole qui contribue au Produit Intérieur Brut (PIB) à plus de 43\% (Banque Africaine de Développement, 2018). Ce secteur représente le premier générateur de revenu en occupant plus de $80 \%$ de la population active. Aussi, les perspectives de lutte contre la pauvreté reposent essentiellement sur l'amélioration de la productivité agricole. Ce système de production majoritairement pluvial et en conditions pédoclimatiques défavorables, s'est souvent montré inefficient ; occasionnant des crises alimentaires récurrentes (WFP, 2010, Abdoul Habou et al., 2016 ; PARM, 2016). Cependant, il existe d'important potentiel en terre irrigable (Ministère de l'Agriculture, 2015 ; Nazoumou et al., 2016) dont la mise en valeur permettrait de contribuer à l'atteinte de la sécurité alimentaire et à la lutte contre la pauvreté (Tillie et al., 2019), réduisant ainsi la dépendance de la production vis-à-vis des aléas climatiques. Ainsi plusieurs formes d'irrigation ont été développées.

Les plus importantes formes d'irrigation en termes de superficie sont les aménagements hydroagricoles à grande échelle (Ehrnrooth et al., 2011). Cependant, du fait de la faible rentabilité et des problèmes de gestion de ces aménagements durant ces dernières années, une attention particulière a été accordée à la petite irrigation (Banque Mondiale, 2009, Ministère de l'Agriculture, 2015). Cette dernière présente l'avantage d'avoir un coût d'investissement très faible et des infrastructures physiques beaucoup plus simples et ayant des entretiens moins onéreux et facilement assimilables par les producteurs. Par ailleurs, il existe un certain nombre de défis à relever dont l'amélioration de la productivité agricole qui faciliterait la rentabilisation économique des investissements. Ce qui implique, l'optimisation des ressources et la minimisation des coûts de production dont le plus important en irrigation est celui de la consommation d'énergie pour assurer la 
mobilisation de l'eau (Belaud et al., 2020 ; Rocamora et al., 2013, Shah et al., 2018).

En milieu rural isolé des installations électriques, diverses alternatives de source d'énergie sont recherchées et utilisées pour la mobilisation et la distribution des eaux d'irrigation (Carrillo-Cobo et al., 2014 ; Chandel et al., 2015 ; Wydra et al., 2019a; Wydra et al., 2019b). Au nombre de ces alternatives, figurent l'énergie thermique à base des carburants fossiles, l'énergie photovoltaïque, l'énergie éolienne et celle de la biomasse (Isaias et al., 2019, Shinde et Wandre, 2015 ; Ibrik, 2020). A titre illustratif, la plupart des producteurs du périmètre irrigué de Soumarana (dans la région de Maradi à l'Est du Niger), utilisaient auparavant les pompes thermiques à essence. Grâce à l'appui financier de leurs partenaires, il leur a été installé un certain nombre de pompes immergées équipées de panneaux solaires, pour la mobilisation et la distribution de l'eau d'irrigation. Ces nouvelles installations ont pour objectifs d'améliorer la productivité des systèmes de production et les rendre plus durables. Le pompage de l'eau à l'aide de l'énergie solaire photovoltaïque ou système de pompage photovoltaïque (SPPV), comparativement au système de pompage thermique (SPT), présente comme avantage la réduction des émissions de gaz à effet de serre, un coût de maintenance réduit, une longue vie utile et l'énergie solaire est inépuisable (Shinde et Wandre, 2015; Hartung et Pluschke 2018). Toutefois, son inconvénient est d'avoir un coût d'investissement initial élevé. Ce qui, limite son utilisation par les producteurs (Wydra et al.,2019a ; Wydra et al., 2019b). Au périmètre irrigué de Soumarana, après l'installation des systèmes de pompage solaire, il s'en est suivi la nécessité d'évaluer la rentabilité des investissements réalisés pour justifier la promotion de cette technologie. C'est dans ce cadre que la présente étude a été menée. Elle a consisté à comparer les systèmes de pompage solaire et thermique, afin de déterminer le système le plus productif du point de vue de la rentabilité économique.

\section{Matériel et méthode \\ 2.1.Présentation de la zone d'étude}

L’étude a été menée dans le périmètre irrigué de Soumarana, situé dans la commune rurale de Safo, (Figure 1). Le village de Soumarana présente un climat du type sahélo-soudanien caractérisé par une pluviométrie moyenne annuelle de $537 \mathrm{~mm}$. Le sol est à prédominance limoneux. La superficie totale du périmètre est estimée à 450 ha dont seulement 250 ha sont mis en valeur par environ 2000 exploitants, repartis dans deux organisations paysannes (OP). La taille d'une exploitation individuelle varie entre 0,15 et $0,5 \mathrm{ha}$. La profondeur de la nappe se caractérise par un niveau statique variant entre 8 et $10 \mathrm{~m}$ selon la période de l'année. L'exhaure de l'eau est assurée par deux types de systèmes de pompage à savoir le système de pompage photovoltaïque 
(SPPV) et le système de pompage thermique (SPT), avec chacun une capacité de $7 \mathrm{~m}^{3} / \mathrm{h}$ dimensionnés pour irriguer une superficie de $0,8 \mathrm{ha}$.

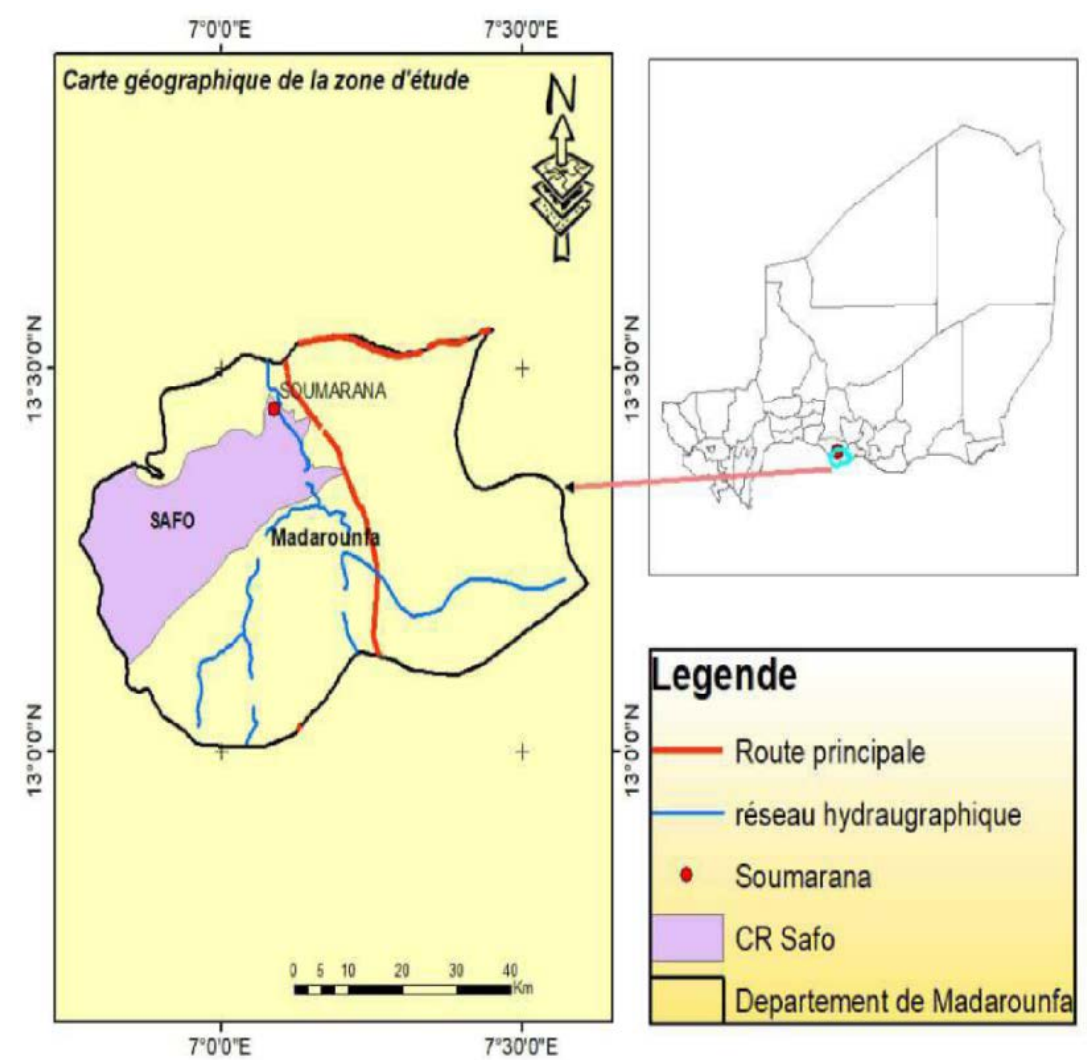

Figure 1. Carte géographique du village de Soumarana. Source : Auteurs, 2021

\subsection{Méthodologie}

La méthodologie repose sur une enquête technico-économique de deux systèmes de pompage en place. Ainsi, 80 exploitants choisis de manière aléatoire du périmètre ont été enquêtés à raison de 40 par type de système de pompage. Les données collectées portent sur la taille d'exploitation par producteur, les principales cultures pratiquées, les charges liées aux intrants (semences, produits phytosanitaires, engrais, carburant), à la main d'œuvre et à l'entretien du système. De même, les informations sur les paramètres d'arrosage (durée et fréquence d'arrosage), la production et le prix de vente des produits, etc., ont été collectées. Les données relatives aux coûts d'investissements initiaux et la durée de vie des différents équipements de systèmes de pompage ont été reçues auprès des l'entreprises ayant fourni ces équipements (Tableau 1).

Tableau 1. Coût d'investissement initial et durée de vie des systèmes de pompage 


\begin{tabular}{ccc}
\hline Désignation & Investissement Initial (FCFA) & Durée de vie (an) \\
Générateur photovoltaïque & 480000 & 20 \\
Electropompe & 800000 & 7 \\
Accessoire & 200000 & 10 \\
Expertise génie civil & 200000 & 20 \\
Ouvrage & 120000 & 20 \\
\hline & SPT & \\
\hline Désignation & Investissement Initial (FCFA) & Durée de vie (an) \\
Moto pompe & 225000 & 4 \\
Abri & 4000 & 1 \\
Ouvrage & 120000 & 20 \\
\hline
\end{tabular}

Source : Entreprise ELYFROS Maradi

Microsoft Excel (version 2019) a été utilisé pour le traitement des données. Le compte d'exploitation des principales cultures par exploitants, et la rentabilité économique des différents systèmes de pompage à travers l'analyse du coût du mètre cube d'eau pompé (CMEP) ont été évalués selon les méthodes de Bouzidi et al. (2006) et Ibrik (2020). La marge brute de production (MBP) des cultures a été déterminée suivant la formule utilisée par Maiga Djibril (2021) et Hossain et al., (2015). Le CMEP a été calculé selon la formule ci-dessous. Il est le rapport entre le coût d'exploitation total annuel (CETA) et le volume d'eau pompé annuellement (VEPA).

$$
\operatorname{CMEP}\left(\frac{F C F A}{m^{3}}\right)=\frac{C E T A(F C F A)}{\operatorname{VEPA}\left(\mathrm{m}^{3}\right)}
$$

La CETA représente la somme des coûts de fonctionnement annuel (CFA) du système de pompage et l'amortissement total annuel des équipements (ATA). Le CFA comporte les frais d'entretien, de gardiennage et éventuellement la charge liée à la consommation de l'essence pour le système de pompage thermique. L'amortissement des équipements a été calculé en appliquant la formule suivante (Bouzidi et al., 2006) :

Où :

$$
A T A(F C F A)=\frac{T * I}{\left[1-(1+T)^{-n}\right]}
$$

$\mathrm{T}(\%)$ : taux d'amortissement,

$\mathrm{n}$ : durée de vie de l'équipement (ans),

$\mathrm{I}$ : investissement initial.

Il a été considéré un taux d'amortissement annuel linéaire défini comme l'inverse de la durée de vie de l'équipement.

Pour le cas du SPT, la variation du CMEP a été simulée en fonction du prix de l'essence, en considérant les autres composantes fixes (charge d'entretien et vidange et l'amortissement total annuel du système), dans l'objectif de déterminer la valeur du prix du litre de l'essence pour lequel le CMEP est le même pour les deux systèmes de pompage. 
La marge brute de production (MBP) est la différence entre la valeur monétaire de la production (VMP) et la charge totale de la production (CTP), qui elle-même comporte les différentes charges variables (intrants, main d'œuvre, entretien des équipements).

$$
M B P(F A C F A / h a)=V M P(F C F A / h a)-C T P(F C F A / h a)
$$

La valeur monétaire de la production est le produit entre la quantité de la production et son prix au moment de la récolte.

\section{Résultats et discussion}

\subsection{Analyse du compte d'exploitation}

Le Tableau 2 présente, les valeurs moyennes des composantes des comptes d'exploitations des principales cultures (carotte et oignon), par type de système de pompage. Il s'agit d'une part, des charges variables liées à l'achat des semences, des engrais chimique et organique, des traitements phytosanitaires, de la main d'œuvre, des entretiens et de l'essence, et d'autre part, de la valeur monétaire de la production de chacune des cultures. Ces valeurs dépendent beaucoup de la spéculation des prix au moment de l'étude, toutes fois, elles permettent d'établir une comparaison de la performance entre les deux systèmes de pompages.

L'analyse du Tableau 2 indique que les valeurs des charges ont varié de manière diverse due à la spéculation des prix au moment de l'étude, comme le montre les coefficients de variation (CV) qui ont oscillé entre 9,58 et $31,20 \%$. Par ailleurs, une comparaison des valeurs des composantes de la CTP de cultures selon le système de pompage montre une légère variation, sauf pour le cas des charges dues à l'achat de l'essence et/ou à l'entretien qui sont plus importantes dans le SPT comparativement à ceux du SPPV. Pour le cas du SPT les charges de l'essence et entretien représentent $40,71 \%$ et $46,22 \%$ de la CTP respectivement pour la carotte et l'oignon, contre $15,40 \%$ et $25,46 \%$ dans le SPPV qui se limitent uniquement aux charges d'entretien du système. Ces résultats sont proches de ceux trouvés par Andres et al. (2016) dans cette même zone d'étude, où la charge due à l'achat de carburant a représenté à elle seule $44 \%$ des charges totales de production dans un SPT. Par contre, ils sont supérieurs à ceux de producteurs de poivron dans la région de Diffa avec en moyenne 32\% de charges opérationnelles à l'hectare (Dalla et al. 2016). Cette forte charge due à la consommation de l'essence et/ou à l'entretien du SPT justifierait la supériorité de la charge totale dans ledit système vis-à-vis de celle du SPPV. Ce qui a conduit à des valeurs de la MBP plus élevées en SPPV pour les deux cultures avec des différences de l'ordre de 402148 FCFA/ha et 342812 FCFA/ha respectivement pour la culture de carotte et de l'oignon. En effet les valeurs de MBP des cultures en système thermique équivalent à 71 et $83 \%$ (respectivement pour la carotte et l'oignon) de celles du système de pompage soaire. 
ESJ Natural/Life/Medical Sciences

\begin{tabular}{|c|c|c|c|c|c|c|c|c|c|}
\hline Culture & $\begin{array}{c}\text { Semences } \\
\text { (FCFA/ha } \\
\text { ) }\end{array}$ & $\begin{array}{c}\text { Main } \\
\text { d'ouvre } \\
\text { (FCFA/ha } \\
\text { ) }\end{array}$ & $\begin{array}{c}\text { Engrais } \\
\text { chimique } \\
\text { (FCFA/ha } \\
\text { ) }\end{array}$ & $\begin{array}{c}\text { Engrais } \\
\text { organique } \\
\text { (FCFA/ha } \\
\text { ) }\end{array}$ & $\begin{array}{c}\text { Traitements } \\
\text { phytosanitaire } \\
\text { s (FCFA/ha) }\end{array}$ & $\begin{array}{c}\text { Entretien } \\
\text { et/ou essence } \\
\text { (FCFA/ha) }\end{array}$ & $\begin{array}{c}\text { CTP } \\
\text { (FCFA/ha } \\
\text { ) }\end{array}$ & $\begin{array}{c}\text { VMP } \\
\text { (FCFA/ha } \\
\text { ) }\end{array}$ & $\begin{array}{c}\text { MBP } \\
\text { (FCFA/ha } \\
\text { ) }\end{array}$ \\
\hline \multicolumn{10}{|c|}{ SPPV } \\
\hline Carotte & 134063 & 183538 & 77150 & 47820 & 39076 & 103231 & 584877 & 1971724 & 1386847 \\
\hline $\mathrm{CV}$ & $31,20 \%$ & $26,75 \%$ & $23,10 \%$ & $20,01 \%$ & $14,31 \%$ & $15,40 \%$ & $27,33 \%$ & $23,45 \%$ & $28,17 \%$ \\
\hline \% de CTP & $23 \%$ & $31 \%$ & $13 \%$ & $8 \%$ & $7 \%$ & $18 \%$ & $100 \%$ & - & - \\
\hline $\begin{array}{l}\text { Oignon } \\
\text { bulbe }\end{array}$ & 96543 & 197779 & 110842 & 52964 & 16780 & 162231 & 637138 & 2580586 & 1943448 \\
\hline $\mathrm{CV}$ & $15,00 \%$ & $27,31 \%$ & $18,40 \%$ & $17,07 \%$ & $25,21 \%$ & $12,32 \%$ & $20,52 \%$ & $30,10 \%$ & $19,74 \%$ \\
\hline \% de CTP & $15 \%$ & $31 \%$ & $17 \%$ & $8 \%$ & $3 \%$ & $26 \%$ & $100 \%$ & - & - \\
\hline \multicolumn{10}{|c|}{ SPT } \\
\hline Carotte & 161364 & 189585 & 107400 & 35412 & 44917 & 369920 & 908597 & 1893296 & 984699 \\
\hline $\mathrm{CV}$ & $17,20 \%$ & $10,89 \%$ & $21,18 \%$ & $9,58 \%$ & $24,35 \%$ & $19,23 \%$ & $29,40 \%$ & $15,41 \%$ & $22,13 \%$ \\
\hline \% de CTP & $18 \%$ & $21 \%$ & $12 \%$ & $4 \%$ & $5 \%$ & $40 \%$ & $100 \%$ & - & - \\
\hline $\begin{array}{l}\text { Oignon } \\
\text { bulbe }\end{array}$ & 100077 & 186686 & 119989 & 36122 & 13267 & 392031 & 848172 & 2448808 & 1600636 \\
\hline $\mathrm{CV}$ & $25,10 \%$ & $10,25 \%$ & $16,11 \%$ & $13,15 \%$ & $19,80 \%$ & $27,12 \%$ & $23,10 \%$ & $14,47 \%$ & $23,10 \%$ \\
\hline \% de CTP & $12 \%$ & $22 \%$ & $14 \%$ & $4 \%$ & $2 \%$ & $46 \%$ & $100 \%$ & - & - \\
\hline
\end{tabular}




\section{ESJ Natural/Life/Medical Sciences}

\subsection{Analyse du coût du mètre cube d'eau pompé}

Le Tableau 3 présente les valeurs de l'amortissement annuel et la charge de fonctionnement annuelle des différents systèmes de pompage pour la mobilisation d'un volume d'eau équivalent à $7350 \mathrm{~m}^{3}$ correspondant au volume d'eau pompé annuellement par les deux systèmes ; étant donné que les caractéristiques des pompes sont identiques pour les deux types de systèmes de pompage et de même pour les valeurs estimées du temps de pompage.

Tableau 3. Valeurs des paramètres de calcul du coût du mètre cube d'eau pompé

\begin{tabular}{ccc}
\hline Libellé & SPPV & SPT \\
\hline ATA (FCFA) & 284928 & 108903 \\
CFA (FCFA) & 136017 & 484585 \\
CV (\%) & 28 & 13 \\
CETA (FCFA) & 420945,13 & 593489 \\
Volume d'eau annuel $\left(\mathrm{m}^{3}\right)$ & 7350 & 7350 \\
CMEP (FCFA $\left./ \mathrm{m}^{3}\right)$ & 57,27 & 80,75 \\
\hline
\end{tabular}

Il ressort de l'analyse de ce tableau que le montant d'amortissement total annuel du système de pompage thermique ne représente que 38,22\% de celui du pompage solaire. Ceci corrobore les résultats de nombreuses études qui ont souligné le coût élevé des investissements initiaux pour l'acquisition des SPPV (Sonou et Abric, 2010 ; Maurya et al., 2015 ; Hossain, 2015 ; Aliyu et al., 2018 ; Shouman et al., 2018 ; Deli et al., 2018). Cela est souvent considéré comme une contrainte qui rend difficile l'accessibilité de ces systèmes de pompage photovoltaïque aux petits producteurs (Wydra et al., 2019a ; Wydra et al., 2019b ; Diop et al., 2020 ; Wazed et al., 2018). Par ailleurs, les CMEP obtenus sont similaires à ceux de Nonbondieu et al. (2018) mais supérieurs à ceux de Ibrik (2020) pour le cas du SPPV. Par contre, pour le SPT, les CMEP sont nettement inférieurs à ceux présentés par World Bank (2018) et ceux de Ibrik (2020). Toutefois, les CMEP du SPT sont plus élevés que ceux du SPPV bien que son coût d'investissement initial soit le plus faible. Cela se justifie par la très faible charge de fonctionnement annuelle du système solaire qui a correspondu à seulement $28 \%$ de celle du système thermique, conduisant ainsi à une charge d'exploitation totale annuelle faible (soit $71 \%$ de celle du système thermique). Ce qui est en harmonie avec les résultats obtenus par Shinde et Wandre (2015) et Ibrik (2020).

La Figure 2 présente la variation des valeurs simulées du CMEP du système de pompage thermique en fonction du coût de l'essence. 


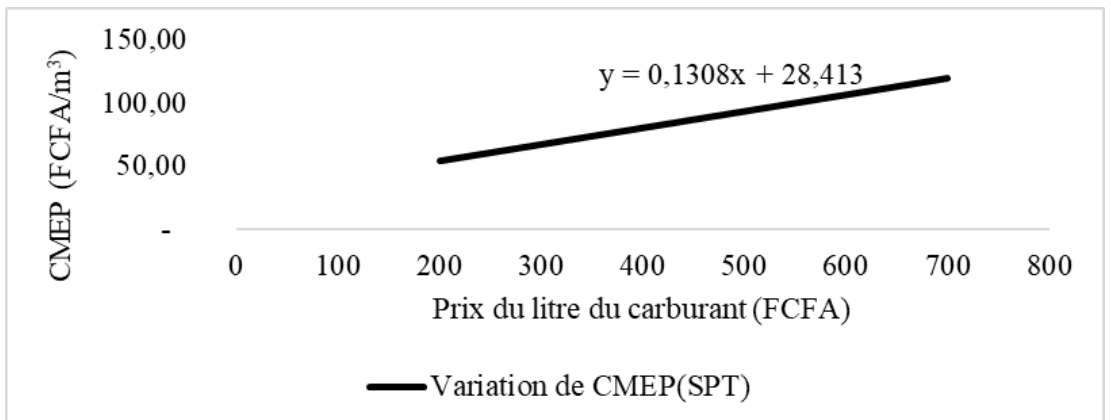

Figure 2. Valeurs simulées du CMEP avec le SPT en fonction du prix de l'essence

On observe que le CMEP s'accroit avec l'augmentation du prix de l'essence. Par ailleurs, on constate que le CMEP du SPT correspond à celui du SPPV seulement si le prix du litre de l'essence équivaudrait à 220,63 FCFA, ce qui est très loin des réalités du marché (minimum 250 FCFA le litre sur le « marché noir »).

\section{Conclusion}

Cette étude présente les performances du système de pompage solaire comparativement à celles du pompage thermique du périmètre irrigué de Soumarana au Niger. La marge brute des principales cultures a été meilleure en système de pompage solaire. De même, malgré son coût d'investissement initial plus élevé, le pompage solaire s'est avéré moins cher, avec un coût du pompage du mètre cube d'eau de seulement $71 \%$ de celui du système de pompage thermique. Les équipements de pompage solaire pourraient de ce fait constituer une bonne alternative aux producteurs dudit périmètre irrigué. Cela est aussi valable pour l'ensemble de la zone du Sahel où les coûts liés à la consommation d'énergie pour la mobilisation et la distribution de l'eau d'irrigation sont l'une des principales charges de production. Ces charges sont difficilement supportables et constituent un des facteurs limitants à l'essor des cultures irriguées notamment au Sahel avec des producteurs à faible capacité financière. Elles conditionnent la rentabilité et la durabilité des systèmes de production irriguées. Toutefois, une étude technico économique plus poussée doit être conduite afin d'approfondir les résultats.

\section{Conflit d'intérêt}

Les auteurs déclarent qu'il n'existe pas de conflits d'intérêts entre eux ni par rapport à aucune information de cette étude

\section{References:}

1. Abdoul Habou, AZ., Boubacar, MK, Abam, T. (2016). Les systèmes de production agricoles du Niger face au changement climatique : défis 
et perspectives. International Journal of Biological and Chemical

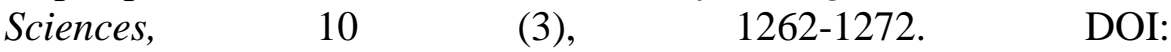
http://dx.doi.org/10.4314/ijbcs.v10i3.

2. Aliyu, M., Hassan, G, Said, SA, Siddiqui, MU, Alawami, AT et Elamin, I.M. (2018). A review of solar-powered water pumping systems. Renewable and Sustainable Energy Reviews, 87, 61-76. https://doi.org/10.1016/j.rser.2018.02.010.

3. Andres, L., Dambo, L, Bode, S, Babousouna, A, Populin, M, Yamba, B et Lebailly, P. (2019). Irrigated food system in Maradi, Republic of Niger: A compararison between operating accounts. VII International Scientific Agriculture Symposium, Jahorina october 06-09, 2019. 2763-2767

4. Banque Africaine de Développement. (2018). Perspectives économiques en Afrique de l'Ouest. https://www.afdb.org/fileadmin/uploads/afdb/Documents/Publication s/2018AEO/Perspectives_economiques_en_Afrique_2018_Afrique_d e_l_Ouest.pdf

5. Banque mondiale. (2009). Développement de l'irrigation au Niger, Diagnostics et options stratégiques, Washington DC, 139p. http://documents.banquemondiale.org/curated/fr/5676114680965728 99/pdf/493790FRENCH0p1ent0irrigation0niger.pdf

6. Belaud, G., Mateos, L, Aliod, R, Buisson, MC, Faci, E, Gendre, S, Ghinassi, G, Gonzales Perea, R, Lejars, C, Maruejols, F, et Zapata, N. (2020) IRRIGATION AND ENERGY: ISSUES AND CHALLENGES. Irrig. and Drain, 69 (S1), 177- 185). https://doi.org/10.1002/ird.2343.

7. Bouzidi, B., Malek, A et Haddadi, M. (2006). Rentabilité économique des systèmes de pompage photovoltaïques. Revue des Energies $\begin{array}{llllll}\text { Renouvelables, } & 9 & \text { (3), } & 187 & - & 197 .\end{array}$ https://www.asjp.cerist.dz/en/article/118188

8. Carrillo-Cobo, M., Camacho-Poyato, E, Montesinos, P et Rodríguez Díaz, J. (2014). Assessing the potential of solar energy in pressurized irrigation networks. The case of Bembézar MI irrigation district (Spain). Spanish Journal of Agricultural Research, 12 (3), 838-849. doi:http://dx.doi.org/10.5424/sjar/2014123-5327

9. Chandel, S.S., Naik, MN et Chandel, R. K. (2015). Review of solar photovoltaic water pumping system technology for irrigation and community drinking water supplies. Renewable \& Sustainable Energy Reviews, 49, 1084-1099.

10. Dalla K., Abdoua M., Maman Abdou M., Guéro M., Ibrahima Inkaye O. et Patrick D. (2016). Résultats du conseil de gestion à l'exploitation 
agricole pour la culture du poivron / campagne 2015 - 2016, Partie (1). Chambre Regionale Agriculture de Diffa. Niger.

11. Deli, K., Djongyang, N, Njomo, D et Tamba, J. (2018) "Photovoltaic water pumping systems as alternative to gasoline water pumping systems in agriculture in Cameroon: CO2 emissions assessment”, Journal of Renewable Energies, 21(2), 279-294. Available at: https://revue.cder.dz/index.php/rer/article/view/688

12. Diop, L., Sar, A, Diatta, I, Wane, YD, Diallo, DM, Bobian, A, Seck, MS, Mateos, L et Lamaddalena, N. (2020). TECHNICAL AND ECONOMIC FEASIBILITY OF SOLAR IRRIGATION PUMPING SYSTEM: A REVIEW. Knowledge-Based Engineering and Sciences, 1 (01), 1-22. https://doi.org/10.51526/kbes.2020.1.01.1-22

13. Ehrnrooth, A. Dambo, L. Jaubert, R. (2011). Projets et programmes de développement de l'irrigation au Niger (1960-2010) : Eléments pour un bilan. Centre d'Etude et d'Information sur la Petite Irrigation (CEIPI). 115p.

14. Hartung, H., et Pluschke, L. (2018). The Benefits and Risks of SolarPowered Irrigation-A Global Review. Rome, Italy. Food and Agriculture Organization of the United Nations and Deutsche Gesellschaft für Internationale Zusammenarbeit. https://www.fao.org/3/I9047EN/i9047en.pdf

15. Hossain, M.A., Hassan, MS, Mottalib, MA et Ahmed, S. 2015. Technical and economic feasibility of solar pump irrigations for ecofriendly environment. Procedia Engineering, 105, 670 - 678. https://doi.org/10.1016/j.proeng.2015.05.047

16. Ibrik, I.H. (2020).Techno-economic Feasibility of Energy Supply of Water Pumping in Palestine by Photovoltaic-systems, Diesel Generators and Electric Grid. International Journal of Energy Economics and Policy, 10 (3), 69-75. DOI: https://doi.org/10.32479/ijeep.8816

17. Isaías, D.H., Cuamba, BC, Leão, A. J. (2019). A Review on Renewable Energy Systems for Irrigation in Arid and Semi-Arid Regions. Journal of Power and Energy Engineering, 07, 21-58.

18. Maïga Djibril I.G. et Boubacar S. (2021). Analyse de la Rentabilité Économique du Maraîchage d'hivernage dans les Communes d'Imanan et de Tagazar au Niger. European Scientific Journal, ESJ, 17(17), 362. https://doi.org/10.19044/esj.2021.v17n17p362

19. Maurya, V.N., Ogubazghi, G, Misra, BP, Maurya, AK et Arora, D.K. (2015). Scope and Review of Photovoltaic Solar Water Pumping System as a Sustainable Solution Enhancing Water Use Efficiency in Irrigation. American Journal of Biological and Environmental Statistics, 1 (1), 1-8. doi: 10.11648/j.ajbes.20150101.11 
20. Ministère de l'Agriculture. (2015). Stratégie de la petite irrigation au Niger (SPIN).

https://recaniger.org/IMG/pdf/SPIN_FINALE_Niger.pdf

21. Nazoumou, Y. Favreau, G. Adamou, M. M. et Maïnassara, I. (2016). La petite irrigation par les eaux souterraines, une solution durable contre la pauvreté et les crises alimentaires au Niger. Cahiers Agricultures (vol. 25, No 1). https://doi.org/10.1051/cagri/2016005

22. Noubondieu, S., Flammini, A et Bracco, S. (2018). Costs and benefits of solar irrigation systems in Senegal. Dakar, FAO. 28 pp. Licence: CC BY-NC-SA 3.0

IGO. https://www.fao.org/documents/card/es/c/CA2209EN/

23. PARM. (2016). Evaluation des risques agricoles. Focus sur l'accès des petits producteurs aux services financiers, aux marchés et à l'information. Rapport Final. https://p4arm.org/app/uploads/2017/03/niger_risk-assessmentstudy_full-report.pdf.

24. Rocamora, C., Vera, J, et Adadía, R. (2013). Strategy for Efficient Energy Management to solve energy problems in modernized irrigation: analysis of the Spanish case. Irrigation Science, 31(5), 1139-1158. https://doi.org/10.1007/s00271-012-0394-5

25. Shah T., Rajan A, Rai GP, Verma, S et Durga, N. (2018). Solar pumps and South Asia's energy-groundwater nexus: exploring implications and reimagining its future. Environ. Res. Lett, 13 (11). https://doi.org/10.1088/1748-9326/aae53f

26. Shinde V.B. et Wandre, S.S. (2015). Solar photovoltaic water pumping system for irrigation: A review. African Journal of Agricultural Research, 10 (22), 2267-2273. DOI: 10.5897/AJAR2015.9879

27. Shouman, E.R., Ezz, H et Bakhoum, E.S. (2018). Economic Analysis of the Using of Traditional Fuel and Solar Energy to Power Irrigation Pumps in Egypt. International Journal of Engineering Research in Africa, 38, 87-99. https://doi.org/10.4028/www.scientific.net/jera.38.87

28. Sonou, M. et Abric, S. (2010). Capitalisation d'expériences sur le développement de la petite irrigation privée pour des productions à haute valeur ajoutée en Afrique de l'Ouest. Rapport Final. https://www.pseau.org/outils/ouvrages/arid_fao_iwmi_practica_capit alisation_d_experiences_sur_le_developpement_de_la_petite_irrigati on_privee_pour_des_productions_a_haute_valeur_ajoutee_en_afriqu e_de_l_ouest_2010.pdf

29. Tillie, P., Louhichi, K et Gomez., Y.P.S. (2019). Impacts ex-ante de la Petite Irrigation au Niger : Analyse des effets micro-économiques à l'aide d'un modèle de ménage agricole, EUR 29836 FR, Publications 
Office of the European Union, Luxembourg, 2019, doi:10.2760/70964, JRC115744.

30. Wazed, S. M., Hughes, BR, O’Connor, D et Calautit, J.K. (2018) A review of sustainable solar irrigation systems for Sub-Saharan Africa. Renewable and Sustainable Energy Reviews, 81 (Part 1), 1206-1225. https://doi.org/10.1016/j.rser.2017.08.039

31. WFP. 2010. Chocs et vulnérabilité au Niger : Analyse des données secondaires. Rapport global. Siège social : Via C.G. Viola 68, Parco de Medici, 00148, Rome, Italie. https://reliefweb.int/sites/reliefweb.int/files/resources/94F37FF998FE 15CD852577D60061C898-Rapport_complet.pdf

32. World Bank, 2018. Solar pumping. The Basics. https://documents1.worldbank.org/curated/en/880931517231654485/ pdf/123018-WP-P159391-PUBLIC.pdf

33. Wydra, K., Becker, P et Aulich, H. (2019a). Sustainable solutions for solar energy driven drinking water supply for rural settings in SubSaharan Africa: a case study of Nigeria, J. Photon. Energy, 9(4), doi: 10.1117/1.JPE.9.043106

34. Wydra, K., Jaskolski, M, Wagner, L et Mohamed, E.S. (2019b). Nexus approach to solar technology for energy and water supply for sustainable rural development in Egypt: a review. J. Photon. Energy, 9 (4), doi: 10.1117/1.JPE.9.043108 